\title{
Understanding Mobile Phone Activities via Retrospective Review of Visualizations of Usage Data
}

Yash Bhavnani

Google Inc.

Mountain View CA 94043, USA

yashbhavnani@google.com

\section{Kerry Rodden}

Google Inc.

Mountain View CA 94043, USA

krodden@google.com

Laura Cuozzo Guarnotta

Google Inc.

Mountain View CA 94043, USA

lauracg@google.com
Margaret T. Lynn

Allen Institute for AI

Seattle, WA 98103, USA

maggietlynn@gmail.com

\section{Sara Chizari}

Red Hat Inc.

Raleigh NC 27601, USA

sara.chizari@gmail.com

\section{Laura Granka}

Google Inc.

Mountain View CA 94043, USA

granka@google.com

Permission to make digital or hard copies of all or part of this work for personal or classroom use is granted without fee provided that copies are not made or distributed for profit or commercial advantage and that copies bear this notice and the full citation on the first page. Copyrights for components of this work owned by others than ACM must be honored. Abstracting with credit is permitted. To copy otherwise, or republish, to post on servers or to redistribute to lists, requires prior specific permission

Copyright is held by the Association for Computing Machinery. MobileHCI '17, September 04-07, 2017, Vienna, Austria ACM $978-1-4503-5075-4 / 17 / 09 . . \$ 15.00$ ACM 978-1-4503-5075-4/17/09...\$15.00

\begin{abstract}
It can be very challenging to get an accurate understanding of mobile phone usage because of the difficulty of observing phone activity in a natural setting. We describe a retrospective methodology where participants review visualizations of their logged activity in an interview setting, and our lessons learned in applying this methodology in a study of user goals and journeys on mobile devices across apps.
\end{abstract}

\section{Author Keywords}

Mobile phone usage; visualization; ethno-mining; retrospective cued recall.

\section{ACM Classification Keywords}

H.5.m. Information interfaces and presentation (e.g., $\mathrm{HCI}$ ): Miscellaneous.

\section{Introduction}

The personal, portable, and versatile nature of mobile phones means that they are used in many contexts where unobtrusive observation of natural activity is particularly difficult, including private moments (like reading news in bed), social interactions (such as watching a funny video with a friend while sitting together), and multitasking (like getting navigation 
assistance while driving). This presents a challenge when designing a research study with the goal of understanding some aspect of mobile phone usage. Previous published studies [4] [5] [8] [11] have proposed innovative methods to address these challenges, most notably video recording of activity with wearable cameras, and screen recording.

Our team at Google focuses on understanding users' information needs in more depth than is available in a search query [10]. Our team's past studies have successfully used the experience sampling method (ESM) [7], via the Paco mobile app [13], prompting participants at random intervals to ask about a recent information need. However, we were concerned that with this self reporting method we may miss or undercount certain types of activities that users don't typically remember or report [12]. We wanted to try an alternative or complementary method that ideally would be less obtrusive and more complete. Our intent was to capture user goals and journeys on mobile devices across apps.

We chose a combination of logging of participants' mobile phone usage, plus follow-up interviews that focused on visualizations of their usage. In particular, our method was inspired by:

- Ethno-mining [1] [2], where visual summaries of a participant's own log data are used as a prompt for reflection and discussion about their activity.

- Retrospective cued recall [14] [15] [9], where participants are shown a detailed log of their recent activities and are asked to remember as much as possible about those activities (instead of manually keeping track, as in a diary study).

- Laddering [6], where participants are asked 'why' and intent related questions around their actions to understand user intents, goals and values.

In this case study we describe our methodology in detail, plus what we learned about it from our pilot studies - what worked well, and what we would do differently in the future.

\section{Method Overview}

There were two main phases of the research:

- In Part 1, we focused on how participants use their phones and how phone usage fits into their life. They were interviewed about their daily and weekly routines and any broader goals. To prompt reflection, we introduced summaries of their overall mobile app usage, aggregated over an 8-week period.

- In Part 2, we invited back a subset of the participants from part 1 , who were shown more detailed visualizations representing all of their app usage from a recent 3-day period. They were asked to recall their activities during each active mobile session, and provided associated context and motivation for those activities.

We deliberately chose to follow an arc from general to specific - so that in part 1 we could build some rapport with the participants before showing them detailed data in part 2. This was also helpful to understand general motives (e.g. an interest in hiking) so that specific 
actions or activities could be drilled down upon (e.g. tracking a trail).

\section{Participant Selection}

Because this study was intended as a pilot of the methodology, we chose to interview a relatively small group of participants, with the goal of eliciting rich detail rather than representative statistics. We recruited 15 Android phone users from across the United States, between the ages of 21 and 49 (4 were aged between 21-24, 7 were 25-34 and 4 were 35-49). 8 were male and 7 were female. Half of the participants were employed full time (employment ranged from construction to corporate banks and technology companies). Participants who used their phones for at least an hour a day were sent a survey to assess interest and ability to participate in a study (either in person or remote) and final recruitment was based on ensuring a mix of gender, age, and occupation.

Participants were screened from the Cross Media Panel [16], which is Google's initiative to accurately and comprehensively measure media and consumer behavior across devices. People in a panel are recruited to represent small versions of the Internet population and are paid to be on the panel. The study's participants had previously signed up to the Cross Media Panel to have their mobile app usage

continuously recorded, via downloading a customlogging app. We focused at the app level (e.g. which app was in the foreground at a given time), rather than the details of which actions were performed within an app. Note that a panel with a custom-logging app is not required to conduct a study like this. We have used PACO, another application logging tool, [13] in a subsequent study to get similar data on app usage. Another option besides PACO is the app AWARE [3]

The participants received an incentive in return for downloading and using the logging app, and the participants we chose to interview also received separate (larger) incentives for each interview (standard cash incentive for the first 60 min interview and a larger cash incentive for the second $90 \mathrm{~min}$ interview). As part of the recruitment process, we checked that participants were comfortable with the idea of being asked about their mobile phone usage in detail.

Seven of the participants were local to us (in the San Francisco Bay Area) and were interviewed in person; eight were in other locations across the United States and were interviewed remotely via video conferencing and screen sharing. We started with only local participants on the first day of interviews, to help us get comfortable with the method and to make it easier.

\section{Part I: Overall Activity}

In part 1 , we began the interviews with an initial discussion (for about the first half of each session) with the participant about what they had going on in their lives at that time, and how their phone helped them get things done. We asked about their daily routines, jobs, interests, life goals, and use of technology. We developed a metaphor of the phone being like a toolbox, and told them we were interested in learning which tools they use for which purposes, and also encouraged them to share any frustrations. We also asked if they had a to-do list and how they planned to use their phone to help them accomplish items on the list. 
After covering this general background and developing some rapport, we then introduced graphs of their overall mobile phone activity for the past 8 weeks, based on counting the number of logged interactions. These were put into a slide deck, which was printed on paper for in-person participants. It included:

1. A bar chart of their top $30-40$ apps used (depending on how many apps they use overall). For legibility, Figure 1 is a screenshot of only the top 10 apps for one participant.

2. A bar chart of their activity by day of week.

3. A line chart of their activity by hour of day (in the participant's time zone), e.g. the participant in Figure 2 was most active in the evenings.

4. App-specific day-of-week or hour-of-day charts, for 3-5 apps where we had determined via inspection of the charts that the participant may have a routine involving that app - e.g. the participant in Figure 3 uses Spotify during the morning commute.

To keep the discussion focused on what the graphs were showing at a high level, we deliberately kept the visual representations as simple as possible, for example not giving a specific definition of "activity" (most participants seemed to assume it was based on time spent) and smoothing the lines using b-spline interpolation rather than showing the raw data.
We chose to exclude two main types of apps from the graphs due to their potential sensitivity:

- Dating/hook-up apps (e.g. Tinder, Grindr) for 2 participants.

- Health apps (e.g. period trackers, pregnancy app) for 3 participants. We retained fitness applications (e.g. MyFitnessPal, FitBit).

We asked the participants to reflect on these graphs, e.g. asking how this activity might relate to what they had already told us, and whether it brought up anything new or how the app helped them in their daily life. In our introduction of the data, we made sure to emphasize that the data might not be totally correct for several reasons. Some apps were logged that were not running as a result of a user action so users would not have any recollection of having done it. Also, we wanted to lessen the effect of consistency bias and awkwardness if the graphs showed something contrary to what they had previously mentioned.

All interviews were video-recorded and a moderator and note taker conducted the interviews. Interviews were transcribed, then coded to come up with goals those participants had.

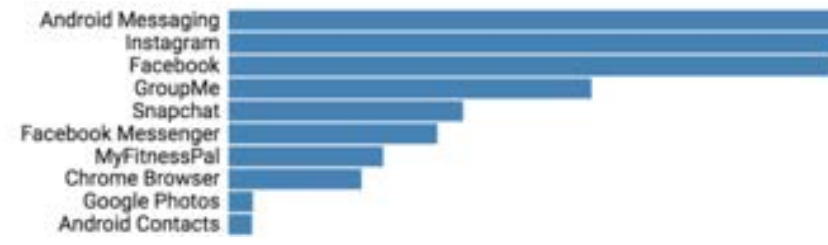

Figure 1: Simple visualization of overall app usage. Illustrative example of the kind of chart we showed participants of their most used apps over an 8-week period. 


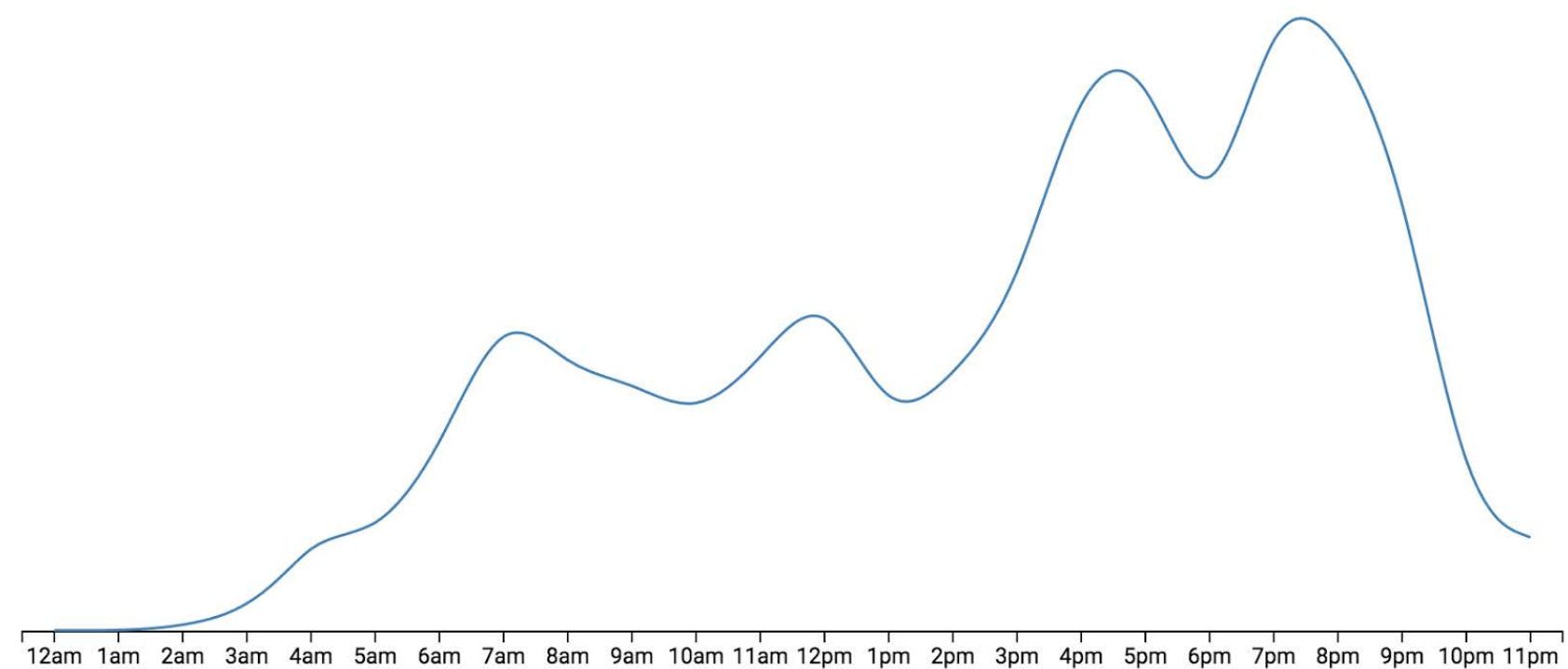

Figure 2: Visualization of a single participant's overall activity by time of day, across an 8-week period, as shown to the participant

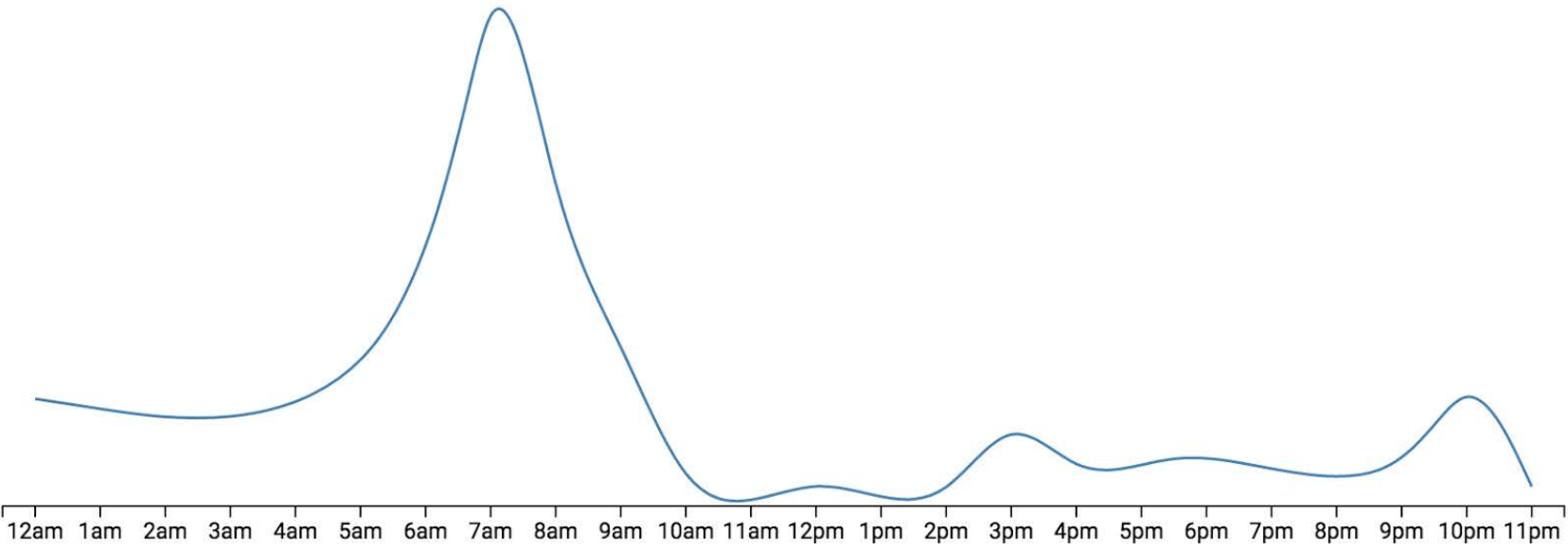

Figure 3: Activity of the same participant as in the previous figure, but for a single app (Spotify), showing a routine during the morning commute 
Lessons learned about the methodology

It was very useful to start off by asking some

background questions about what the participant has going on in their life, what their interests and hobbies are, and any broader goals, before diving into discussion of their mobile usage.

In this initial discussion, participants tended to talk in broad terms, describing many things briefly, so it was useful to write down what they said and come back to each item later to ask for more details and understand why they do these activities and what triggers them for example, to go from "I check LinkedIn" to "I'm looking for a job so I check LinkedIn every day to keep up with news from companies I'm interested in".

We chose to use simple line/bar charts, and these were generally understandable and intuitive to the participants, requiring no explanation beyond the time period represented. In general, the participants thought that the data represented their activity well.

None of the participants expressed any concern in seeing their data represented in this format nor did they express any concerns about privacy or disclosure. Some were intrigued by what they saw (e.g. surprised that they use Facebook so much) as people tended to underestimate the amount of time they spent in socia apps. Some talked about the fact that they were aware of being tracked and had been curious about how the data would be used.

When shown their data, there were a number of instances where participants had not previously recalled some app that they in fact used frequently, so the data was very helpful as a cue. Some participants seemed to feel badly about this discrepancy between their recollection and the logged data. Coupled with the fact that none of the participants seemed bothered by the graphs, we think we could have introduced the data visualizations earlier in the interview.

An alternative methodology for this part would have been to just ask the participants to flip through their phones and talk about the apps they have. Our belief is that it was very helpful to have the data on actual usage, to focus the conversation on most frequent activity, and to prepare app-specific prompts in advance.

\section{Part II: Details of usage}

In part 2, we brought back a subset (7) of the original participants, retaining those with the richest stories around their mobile activities, while trying to maintain diversity in demographics, as well as other aspects such as tech-savviness and affinity for Google products. We had 3 local participants and 4 remote participants.

We created more detailed representations of participants' interactions with their phones, for a recent 3-day period. We called these activity cards, and each card contained a representation of each app used within a single continuous period of phone usage where "continuous" means "with gaps between events that are shorter than 5 minutes". Each app was represented by the app name, inside a rounded rectangle with a fill color chosen from the app's branding or UI (system apps and less popular apps were light grey) - see Figure 4. Below the apps, we showed other text metadata from the activity period, such as searches in Google and web page titles from Chrome. In Figure 4, this participant had previously 
taken a screenshot of a recipe from Chrome, and she referred to that (in Samsung Gallery) while cooking. Here, she wants to record the nutrition information in her calorie counting app, MyFitnessPal. She searches for the recipe again in Chrome so that she can copy and paste the ingredients into MyFitnessPal - something that was not possible with a screenshot. The whole journey is a complex one involving multiple switches between apps to accomplish the goal.

We did not tell the participants in advance exactly what we would be doing, just that we would be looking at their data in more detail than last time. In particular, they did not know which days we would choose to discuss with them. We chose a recent 3-day period that included 2 weekdays and 1 weekend day, depending on availability of data. The biggest gap for any participant was 4 days - in that case, we showed data from the preceding Thursday, Friday, and Saturday for an interview on the Wednesday.

For each activity card we showed participants we asked them to tell us what they were trying to get done, if it was related to a goal they had or part of a routine, if they felt they accomplished what they set out to do and if it was difficult or not. We asked about long and shortterm goals and probed to uncover unmentioned

behavior-driving goals to include activity outside of the mobile phone (e.g. I filled out the job application from my computer).
Lessons learned about the methodology

It was helpful to bring up the goals, journeys, and routines that participants had described in their initial interview, to prompt a discussion of how those related (or did not relate) to the 3 days of detailed data shown in the cards. It was also helpful to probe and relate various activities together to get a complete picture of how a participant completed a journey that may have been broken in various times.

The activity cards helped the participants to recall what they were doing, and none of them seemed to be perturbed by the level of detail we chose to show. In particular, the extra text metadata (e.g. searches and web page titles) was extremely helpful to participants in recalling specific activities or stories. An important area for future work is to investigate the balance between what information is most helpful for recalling activity versus making the participant uncomfortable with the level of detail shown. For example, content of messages in messaging apps might have been a very useful cue for recall - but would probably have felt too intrusive to the participants. When a messaging app was shown on an activity card, the participants would often open their phones and scroll back through messages in order to recall their activities, without having to actually show us their private conversations, and that felt like a comfortable balance. It was also sometimes helpful for them to look through other information on their phones, such as to-do lists and calendars, to help them recall more context for specific activities. 
Sunday December 6 10:54am - 11:04am, 9 minutes 22 seconds
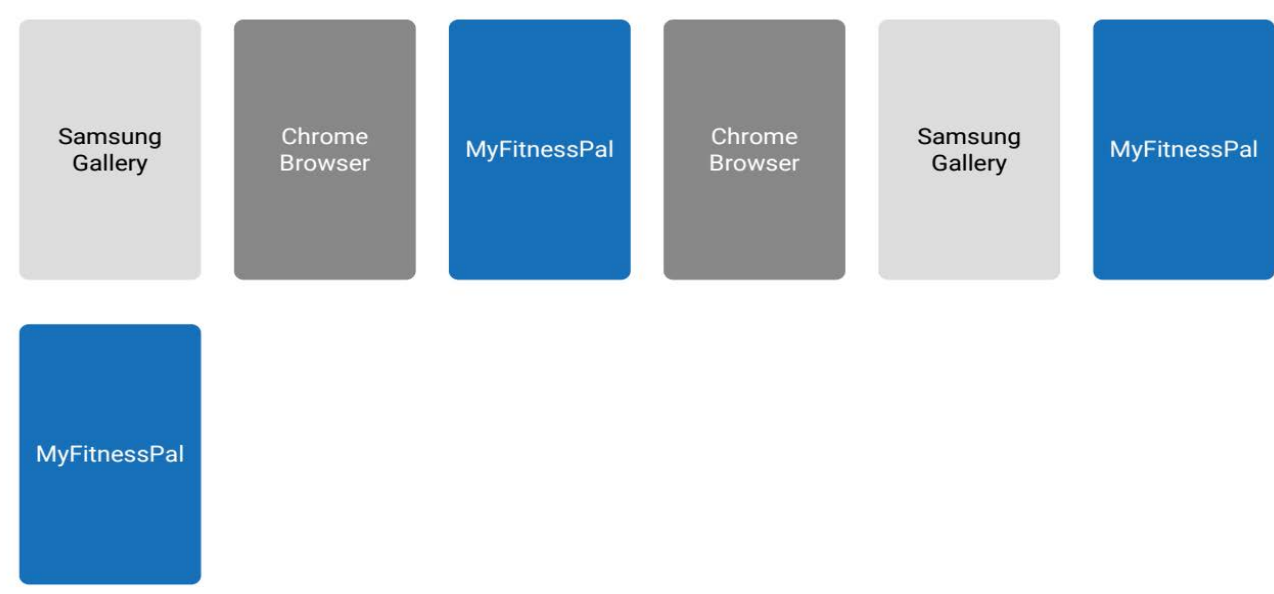

CHROME BROWSER

R

Microwave Mug Cake / Kodiak CakesKodiak Cakes

MYFITNESSPAL

MyFitnessPal

Figure 4: Example activity card, representing each instance of app usage within a single phone session. Showing this to the participant helped elicit her task and associated context

Other types of metadata that we did not log or represent to the participants that would have been potentially useful to try:

- Notifications received, since those are an important trigger to start using the phone. Some participants had apps used only via notifications

(e.g. Google Fit prompts about activity).

- Contacts in messaging apps

- Actions in social media apps (like, post, etc.)

- Screen on/off status
- Coarse location information, e.g. for quick reference of whether the participant was at home, at work, or elsewhere

- Other sensor data, e.g. battery (AWARE [3])

- Screenshots [14] [15] 


\section{Conclusions}

For others who wish to employ this method, we recommend the following best practices:

- Collect data over a long enough period to get data sets representative of routine use

- Conduct a shorter interview initially with each participant to establish rapport. Conduct longer interviews after that, to collect more detailed explanations of activity and motivations

- Create easy-to-understand visuals for users

- Emphasize that data collection is not perfect so participants are less likely to be embarrassed if activity appears in visualizations that they hadn't mentioned

- Use visualizations to help jog memory and assist in remembering

- When asking participants to recall their activity, try to ask about activity that is as recent as possible - ideally within the last 72 hours.

In comparison to an ESM study or a diary study, recalling activity based on logged usage requires no upfront effort on the part of the participant, and is unobtrusive and complete. However, with an ESM or diary study we would probably have been able to collect more context around frequent activities that are hard to distinguish from each other retrospectively (e.g. different Facebook-checking sessions look very similar even when users are doing very different activities with different goals). In the future we might combine logging and real-time self-reporting methods to lessen the reliance on retrospective recall of fleeting activities. However, the keeping of in-situ diary studies might influence the user activities being logged so further investigation is required.

One open question for future work is how to strike the right balance between logging and displaying information that is useful to prompt recall, and making participants uncomfortable with the logging. Another aspect for consideration is having some way for having participants tag or self identify the activities they were doing to avoid extensive analysis of interview data. One possible way to do this would be to use PACO [13] to combine this method with ESM by surveying participants about their activity as it happens.

\section{Acknowledgements}

We are grateful to Jay Runquist for help with designing the visualizations shown to participants, Brian Portacio for help with participant scheduling logistics, Allison Woodruff for feedback on the methodology, Dan Russell for feedback on this paper and Dylan Lorimer for his support in use of the panel.

\section{References}

1. Ryan Aipperspach, Ken Anderson, Paul Aoki, John

F. Canny, Tye Lawrence Rattenbury, Allison Woodruff. 2006. Ethno-Mining: Integrating Numbers and Words from the Ground Up. Electrical Engineering and Computer Sciences University of California at Berkeley Tech Report UCB/EECS2006-125 https://www2.eecs.berkeley.edu/Pubs/TechRpts/20 06/EECS-2006-125.pdf

2. Ken Anderson, Dawn Nafus, Tye Rattenbury, and Ryan Aipperspach. 2009. Numbers have qualities too: experiences with ethno-mining. In Ethnographic Praxis in Industry Conference Proceedings (EPIC 2009), 123-140. 
3. AWARE: Android Mobile Context Instrumentation Framework. Retrieved May 1, 2017 from http://www.awareframework.com/

4. Barry Brown, Moira McGregor, and Eric Laurier. 2013. iPhone in vivo: video analysis of mobile device use. In Proceedings of the SIGCHI Conference on Human Factors in Computing Systems (CHI '13), 1031-1040. http://dx.doi.org/10.1145/2470654.2466132

5. Barry Brown, Moira McGregor, and Donald McMillan. 2014. 100 days of iPhone use: understanding the details of mobile device use. In Proceedings of the 16th International Conference on Human-Computer Interaction with Mobile Devices and Services (MobileHCI '14), 223-232. http://dx.doi.org/10.1145/2628363.2628377

6. Brian F. Blake, Abrafi Saaka, Chris Sidon. 2004. Laddering: A "How to Do It" Manual - with a Note of Caution. Retrieved May 11, 2016 from http://academic.csuohio.edu/cbresearch/papers/Go od\%20PDFs/Laddering_A\%20How\%20to\%20do\%2 0it\%20manual.pdf

7. Mihaly Csikszentmihalyi, Reed Larson. 1983. The Experience Sampling Method. New Directions for Methodology of Social \& Behavioral Science Vol 15, Mar 1983, 41-56.

8. Denzil Ferreira, Jorge Goncalves, Vassilis Kostakos, Louise Barkhuus, and Anind K. Dey. 2014. Contextual experience sampling of mobile application micro-usage. In Proceedings of the 16th International Conference on Human-Computer Interaction with Mobile Devices and Services (MobileHCI '14), 91-100.

http://dx.doi.org/10.1145/2628363.2628367

9. Rachel Jones, Natasa Milic-Frayling, Kerry Rodden, and Alan Blackwell. 2007. Contextual method for the redesign of existing software products. International Journal of Human-Computer Interaction 22, 1-2, 81-101.
10. Steven Levy. 2015. Google's secret study to find out our needs. Retrieved December 21, 2016 from https://backchannel.com/googles-secret-study-tofind-out-our-needs-eba8700263bf

11. Donald McMillan, Moira McGregor, and Barry Brown. 2015. From in the wild to in vivo: Video Analysis of Mobile Device Use. In Proceedings of the 17th International Conference on HumanComputer Interaction with Mobile Devices and Services (MobileHCI '15), 494-503. http://dx.doi.org/10.1145/2785830.2785883

12. Andreas Möller, Matthias Kranz, Barbara Schmid, Luis Roalter, and Stefan Diewald. 2013.

Investigating self-reporting behavior in long-term studies. In Proceedings of the SIGCHI Conference on Human Factors in Computing Systems (CHI '13), 2931-2940. http://dx.doi.org/10.1145/2470654.2481406

13. PACO: The Personal Analytics Companion. Retrieved May 1, 2017 from https://www.pacoapp.com/

14. Daniel M. Russell and Ed H. Chi. 2014. Looking back: retrospective study methods for HCI. In Ways of Knowing in HCI, Judith S. Olson and Wendy A. Kellogg (Eds.). Springer, New York, 373393.

15. Daniel M. Russell and Mike Oren. 2009. Retrospective cued recall: a method for accurately recalling previous user behaviors. In 42nd Hawaii International Conference on System Sciences (HICSS'09), 1-9.

16. U.S Reach Cross Media Panel. Retrieved June 22nd 2017 from https://www.crossmediapanel.com/panels/usreach/ welcome 